\title{
Erratum to: Concentrations and identification of culturable airborne fungi in underground stations of the Seoul metro
}

\author{
Sung Ho Hwang ${ }^{1}$ - Soojin Jang ${ }^{2}$ - Wha Me Park ${ }^{3}$. Jae Bum Park ${ }^{4}$
}

Published online: 23 August 2016

(C) Springer-Verlag Berlin Heidelberg 2016

Erratum to: Environ Sci Pollut Res

DOI 10.1007/s11356-016-7291-z

There was an error in the first author name.

The correct name of the 1st Author is Sung Ho Hwang.

The online version of the original article can be found at http://dx.doi. org/10.1007/s11356-016-7291-z.

\section{Jae Bum Park}

jbpark@ajou.ac.kr

1 National Cancer Control Institute, National Cancer Center, 323

Ilsan-ro, Ilsandong-gu, Goyang-si, Gyeonggi-do, South Korea

2 Institut Pasteur Korea, 16 Daewangpangyo-ro 712 beon-gil, Bundang-gu, Seongnam-si, Gyeonggi-do, South Korea

3 Institute of Environmental and Industrial Medicine, Hanyang University, Seoul, South Korea

4 Department of Occupational and Environmental Medicine, Ajou University, School of Medicine, San 5, Woncheon-dong,

Yeongtong-gu, Suwon, South Korea 